\title{
Distribución, abundancia y reproducción de Opisthonema oglinum (Pisces: Clupeidae) en la plataforma continental del sur del Golfo de México
}

\author{
M. C. García-Abad ' , A. Yáñez-Arancibia 2, P. Sánchez-Gil 2, M. Tapia-García ${ }^{1}$
}

1 Departamento de Hidrobiología, D.C.B.S., Universidad Autónoma Metropolitana-Iztapalapa, Apartado Postal 55-535, México 09340, D. F. Fax 7244738. e-mail mtg @xanum.uam.mx

2 Programa de Ecología, Pesquerías, y Oceanografía del Golfo de México (EPOMEX), Universidad Autónoma de Campeche, Apartado Postal 520, Campeche 24030, México. Fax (981) 659 54. e-mail psanchez@balamku.uacam.mx

Recibido 5-V-1997. Corregido 17-I-1998. Aceptado 25-I-1998.

\begin{abstract}
At present the ecological understanding and evaluation of tropical demersal fish communities have their bases on studies about the biology and ecology of dominant species. The Campeche Sound, in the southern Gulf of Mexico, has more than 300 species in the demersal fish community and 30 are dominants. Among these species, Opisthonema oglinum is dominant by its frequency, distribution and abundance. This work determined the pattern of abundance and distribution of $O$. oglinum, and its reproduction, maturity and recruitment. Fish were collected with a shrimp trawl net during six oceanographic cruises in the Campeche Sound. The cruises correspond with the three characteristic seasons in the area: rainy, "nortes", and dry season. A total of 813 individuals with sizes between 56 and $205 \mathrm{~mm}$ of total length were collected; they weighted $30211.5 \mathrm{~g} . O$. oglinum is a typical pelagic-neritic species, and is estuarine dependent. This species occurs in the inner shelf waters of the Campeche Sound, less than $36 \mathrm{~m}$ deep and mainly in areas influenced by coastal discharges. It also occurs in the Terminos Lagoon (southern Campeche Sound) and occasionally in the riverine systems that drain into the lagoon. The highest biomass and density are on depths lesser than $20 \mathrm{~m}$, in June and August-October. The high density of juveniles mainly determines the higher biomass. Maturity can start at a total length of 126 $\mathrm{mm}$, but the size of first maturity is $135 \mathrm{~mm}$ of total length. Reproduction occurs from May to October with two evident peaks during May and August in depths greater than $18 \mathrm{~m}$. The juveniles are found in the shelf and inside of the Terminos Lagoon. Recruitment is from June to October (rainy season). The visceral and gonadic indices showed that the greatest alimentary activity is from June to August and in November. The condition factor is lowest in June, where the recruitment is highest, and highest in November, after spawning.
\end{abstract}

Key words: By-catch, demersal, fish ecology, fish reproduction, Gulf of Mexico, thread herring, Opisthonema oglinum.

La Sonda de Campeche en el sur del Golfo de México, es una zona de intensa actividad pesquera e industrial, lo cual la coloca en una situación prioritaria en cuanto a necesidades de evaluación ecológica de sus recursos. Actualmente, el entendimiento ecológico de las comunidades de peces demersales de la banda tropical, así como la evaluación de su potencial pesquero se basa en el desarrollo de estudios sobre el conocimiento biológico y ecológico de las especies dominantes en particular, en términos de la estructura de la población, reproducción, crecimiento, alimentación y su relación con los diversos factores físico-ambien- 
tales (Pauly 1982, Yáñez-Arancibia y Sánchez-Gil 1986, Yáñez-Arancibia et al. 1993, 1994, Pauly y Yáñez-Arancibia 1994). En esta región, se han realizado estudios prospectivos y de evaluación cuantitativa de las comunidades de peces demersales, en los últimos 18 años, obteniéndose importantes avances en el conocimiento de estas comunidades de alta diversidad, en más de 300 especies. De éstas especies, 30 han sido detectadas como especies dominantes, con base en que presentan una amplia distribución, alta frecuencia en las capturas y una gran abundancia en número y peso. Entre estas especies importantes se encuentra Opisthonema oglinum (Le Sueur, 1817) (Yáñez-Arancibia y Sánchez-Gil 1986, 1988a y b).

En el Golfo de México, existen antecedentes sobre $O$. oglinum referentes a su distribución y abundancia, mencionándose algunos aspectos sobre su biología e importancia dentro de la comunidad (Darnell et al. 1983, Yáñez-Arancibia y Sánchez-Gil 1986, Darnell y Kleypas 1987). Mientras que al nivel de su potencialidad y parámetros poblacionales ha sido estudiada por Houde (1977), Reintjes (1980) y Houde et al. (1983).

Para otras regiones se ha analizado al nivel de pesquerías y su utilización industrial por Ogawa y Barreto (1979) en el noreste de Brasil; sobre tasas de captura por Harvey (1980) y Grant (1981) en Jamaica; algunos aspectos de su biología y pesquería por Valdés y Sotolongo (1983), y sobre la dieta de la especie, Vasconcelos (1979).

Así, el objetivo de este trabajo es caracterizar el patrón de distribución y abundancia, y determinar áreas de reproducción, maduración, crianza, y reclutamiento para conocer la estructura y dinámica de la población de $O$. oglinum en el área de estudio.

\section{MATERIALES Y MÉTODOS}

La Sonda de Campeche al sur del Golfo de México, está situada entre $18^{\circ}$ a $21^{\circ} \mathrm{N}$. Hay tres estaciones climáticas: lluvias (junio-octubre), nortes (octubre-febrero) y sequías (febrero-mayo). Existen dos hábitats o subsistemas ecológicos diferentes en la plataforma continental, ampliamente descritos por YáñezArancibia y Sánchez-Gil (1988a) y representados como Zona A y Zona B (Fig. 1). La porción oeste o Zona $\mathrm{A}$, frente de la Boca del Carmen (BC) está permanentemente influenciada por aguas dulces y estuarinas. Presenta aguas turbias con transparencias de 7 a $42 \%$ (profundidad de extinción del disco de Secchi en relación con la profundidad de la estación de muestreo), alta concentración de sedimentos suspendidos (25.2 mg/l) (Carranza-Edwards et al. 1993), ausencia de plantas bénticas, sedimentos limoarcillosos (10-60\% de $\mathrm{Ca}$ $\mathrm{CO}_{3}$ ), alto contenido de materia orgánica $(\geq 10 \%)$ y salinidades de 32 a 36 . La porción este o Zona B, enfrente de la Boca de Puerto Real (BPR) es un área marina típica con aguas claras (transparencia de 53 a 99\%), pastos marinos y macroalgas, sedimentos arenosos (70$90 \% \mathrm{CaCO}_{3}$ ), bajo contenido de materia orgánica $(\leq 10 \%)$ y salinidades de 36 a 37 .

Las recolectas se efectuaron en seis cruceros oceanográficos realizados entre los 11 y 76 m de profundidad en junio 1978, agosto 1980, noviembre 1980, julio 1981, octubre, 1981 y marzo 1982 (Fig. 1). Las recolectas fueron diurnas y nocturnas con redes de arrastre comerciales de $18 \mathrm{~m}$ de longitud, $9 \mathrm{~m}$ de abertura de trabajo y malla de $11 / 3$ ". Estos arrastres se realizaron a una velocidad promedio de dos nudos durante 30 min cada uno. Los seis cruceros se distribuyeron temporalmente abarcando las distintas épocas climáticas de la región y para el análisis temporal fueron ordenados con base en una secuencia estacional, asumiéndose como un ciclo anual para el estudio de la especie. Las capturas fueron analizadas de acuerdo a Yáñez-Arancibia y Sánchez-Gil (1988b). Fueron calculados los parámetros poblacionales de densidad, biomasa, longitud promedio y peso promedio, para el área muestreada en cada mes de recolecta y para cada estación de muestreo. 


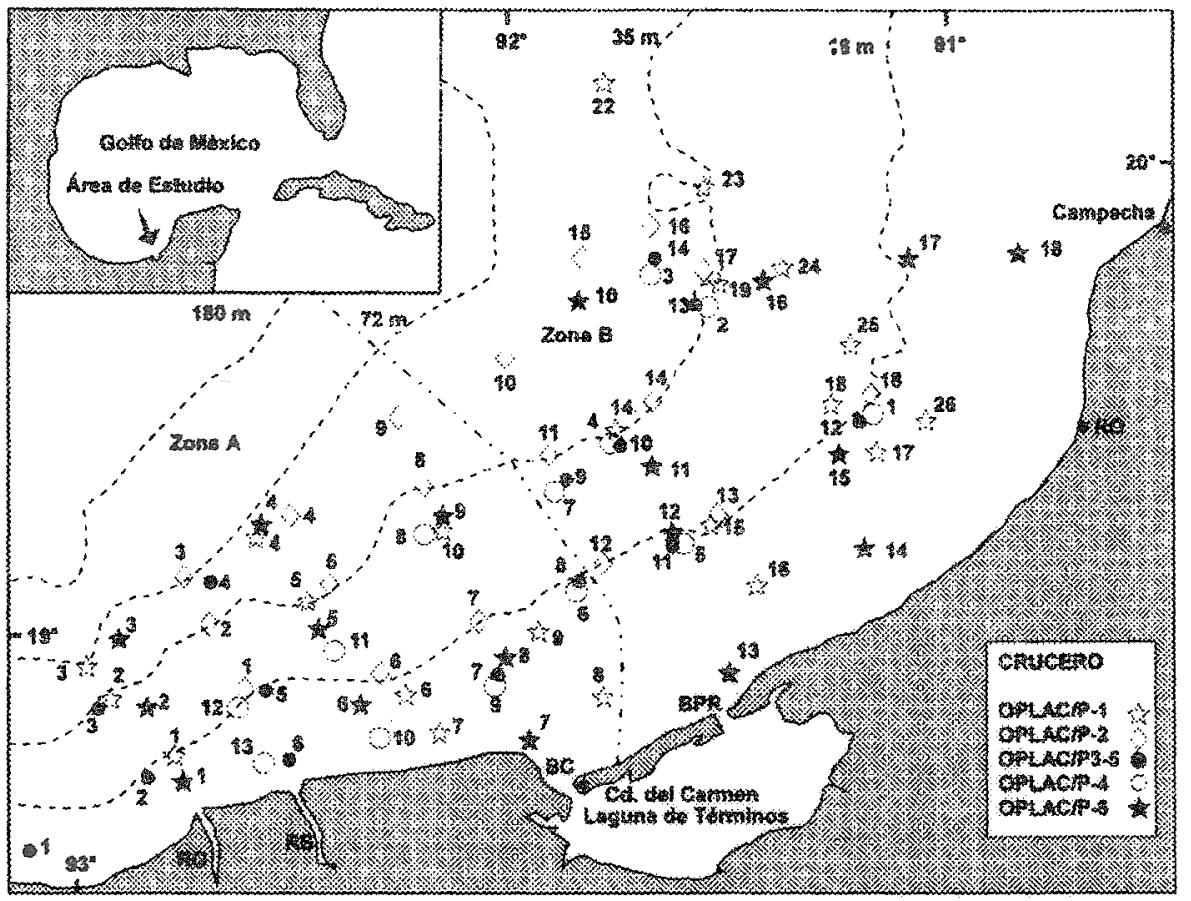

Fig. 1. Sonda de Campeche frente a la Laguna de Términos. Se indica la localización de las estaciones de muestreo de los seis cruceros de investigación realizados. Ríos: Grijalva (RG), Usumacinta (RS) y Champotón (RO). Boca del Carmen (BC) y Boca de Puerto Real (BPR).

\section{Determinación de sexo y madurez goná-} dica: La gónada fue disectada y observada al microscopio para determinar el sexo por su forma, consistencia del tejido y por la presencia o ausencia de huevos. El estado de madurez gonádica se determinó según la escala que presenta Laevastu (1971) en donde fase $\mathrm{I}=$ inmaduros indeterminados sexualmente, II = inmaduros determinados sexualmente, III = en maduración, $\mathrm{IV}=$ maduros, $\mathrm{V}=$ en reproducción, $\mathrm{VI}=$ desovados y VII = adultos en descanso.

Talla de primera madurez: Se tomó como talla de primera madurez el centro de amplitud de las tallas de traslape de los individuos inmaduros (II) y los individuos en maduración (III) (Tapia-García et al. 1995). Por otra parte, se calcularon los porcentajes de machos, hembras e individuos juveniles indeterminados sexualmente con base en la población total y para cada mes de recolecta.
Índice visceral: Fue calculado para la población total, por sexos y por mes según la expresión matemática:

$$
\mathrm{IV}=\mathrm{V} / \mathrm{P}_{\mathrm{T}}
$$

IV = índice visceral, $\mathrm{V}=$ peso de las vísceras y $\mathbb{P}_{\mathrm{T}}=$ peso total.

Índice gonádico: Fue calculado matemáticamente según Cailliet et al. (1986) como:

$$
\mathrm{IG}=\mathrm{G} / \mathrm{P}
$$

$\mathrm{IG}=$ índice gonádico, $\mathrm{G}=$ peso gonádico y $\mathrm{PT}=$ peso total.

Rellación talla/peso: Esta relación es expresada matemáticamente según la ecuación:

$$
\mathrm{P}=\mathrm{aL} \mathbf{b}
$$


que transformada logarítmicamente da un modelo lineal del tipo:

$$
\log _{\mathrm{e}} \mathrm{P}=\log _{\mathrm{e}} \mathrm{a}+\log _{\mathrm{e}} \mathrm{L}
$$

$\mathrm{P}=\operatorname{peso}(\mathrm{g}), \mathrm{L}=$ longitud $(\mathrm{mm}), \mathrm{a}=$ ordenada al origen, $\mathrm{b}=$ pendiente $\mathrm{y} \log _{\mathrm{e}}=\log \mathrm{a}-$ ritmo natural.

Factor de condición: Para conocer el comportamiento de la condición de la población se calculó el factor de condición de Le Cren (1951):

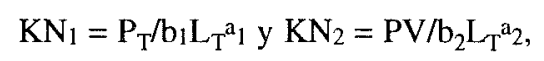

$\mathrm{KN}=$ coeficiente de condición, $\mathrm{b}_{1}=$ factor de condición promedio para $\mathrm{P}_{\mathrm{T}}, \mathrm{b}_{2}=$ factor de condición promedio para $\mathrm{P}_{\mathrm{V}}, \mathrm{a}_{1}=$ coeficiente de alometría para $\mathrm{P}_{\mathrm{T}}, \mathrm{a}_{2}=$ coeficiente de alometría para $\mathrm{P}_{\mathrm{V}}, \mathrm{P}_{\mathrm{T}}=$ peso total, $\mathrm{P}_{\mathrm{V}}=$ peso desviscerado. Los factores de condición promedio y coeficientes de alometría, corresponden a la ordenada al origen y a la pendiente respectivamente, obtenidas de la relación talla/peso tanto para peso total como desviscerado.

\section{RESULTADOS}

Distribución y abundancia espacio-temporal: Fue analizado un total de 813 individuos, con un peso total de $30211.5 \mathrm{~g}$ y tallas entre los 56 y 205 mm (Cuadro 1). Esta especie se distribuye en la plataforma interna de la Sonda de Campeche, entre los 12 y $36 \mathrm{~m}$ de profundidad, tanto en áreas influenciadas por aguas epicontinentales (zona A), como en el área típicamente marina (zona B) (Fig. 2). Espacialmente se observó que durante la época de lluvias y sequías, la mayor biomasa estuvo asociada a las áreas mas someras y de mayor influencia estuarina en la zona A entre 12 y 20 $\mathrm{m}$ de profundidad principalmente. Durante nortes fue más abundante en la zona B (Fig. 3). Asimismo, el análisis mensual permite ver que en junio y en agosto-octubre se notan dos picos

\section{CUADRO}

Abundancia y tallas de Opisthonema oglinum en los
cruceros realizados. Abundance and sizes of Opisthonema oglinum in each cruise.

\begin{tabular}{lrrr} 
Crucero & $\begin{array}{c}\text { Número } \\
\text { (ind.) }\end{array}$ & \multicolumn{1}{c}{$\begin{array}{c}\text { Peso } \\
(\mathrm{g})\end{array}$} & \multicolumn{1}{c}{$\begin{array}{c}\text { Tallas } \\
(\mathrm{mm})\end{array}$} \\
& & & \\
Marzo, 1982 & 33 & 1511.3 & $95-203$ \\
Junio, 1978 & 210 & 3483.0 & $56-205$ \\
Julio, 1981 & 25 & 1168.6 & $160-193$ \\
Agosto, 1980 & 350 & 15921.0 & $100-195$ \\
Octubre, 1980 & 186 & 7754.1 & $85-200$ \\
Noviembre, 1980 & 9 & 373.5 & $95-178$ \\
Captura Total & 813 & 30211.5 & $56-205$
\end{tabular}

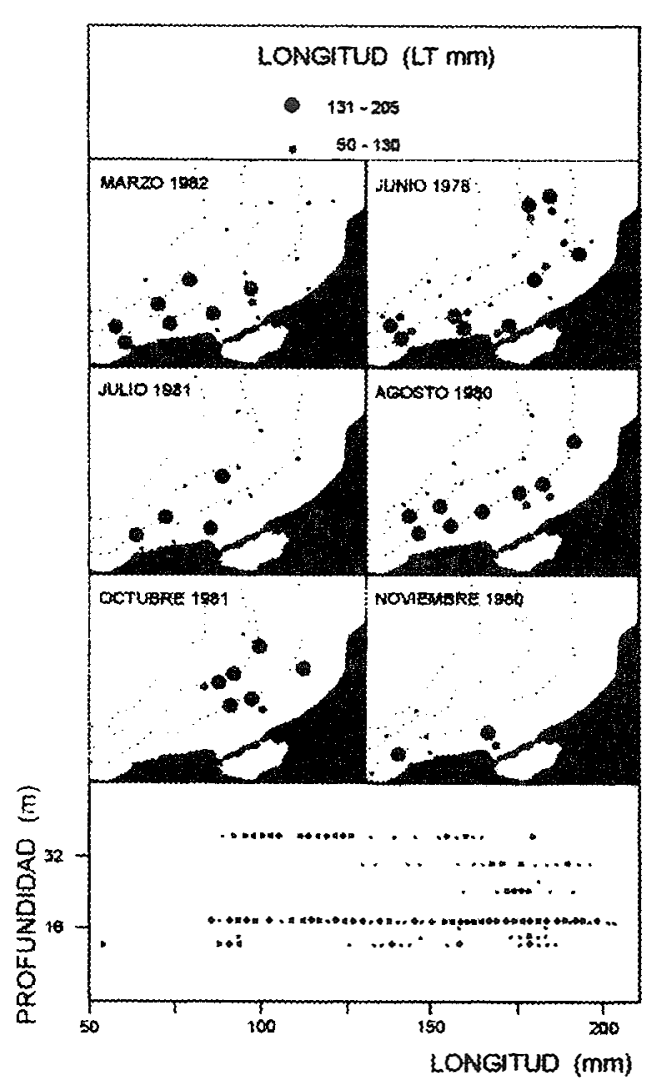

Fig. 2. Distribución de tallas de O. oglinum en los meses analizados. 
en densidad y biomasa (Fig. 3). El primero, se debe a un grupo numeroso de juveniles que se está reclutando a la población adulta y que se puede inferir al observar como se distribuyen por tallas tanto espacial como temporalmente (Figs. 2, 3 y 4). En julio el grupo de juveniles se ha reclutado en su totalidad a la población adulta, caracterizándose por ser pocos individuos con una baja biomasa y tallas grandes
(Figs. 3 y 4). Los altos valores de densidad y biomasa en agosto están caracterizados por individuos en su mayoría de tallas grandes. El máximo pico observado en octubre está dado por un nuevo reclutamiento de juveniles y dos grupos de tallas, uno de individuos pequeños y otro de adultos. En noviembre los bajos valores están dados por un solo grupo pequeño de adultos. (Figs. 3 y 4 ).

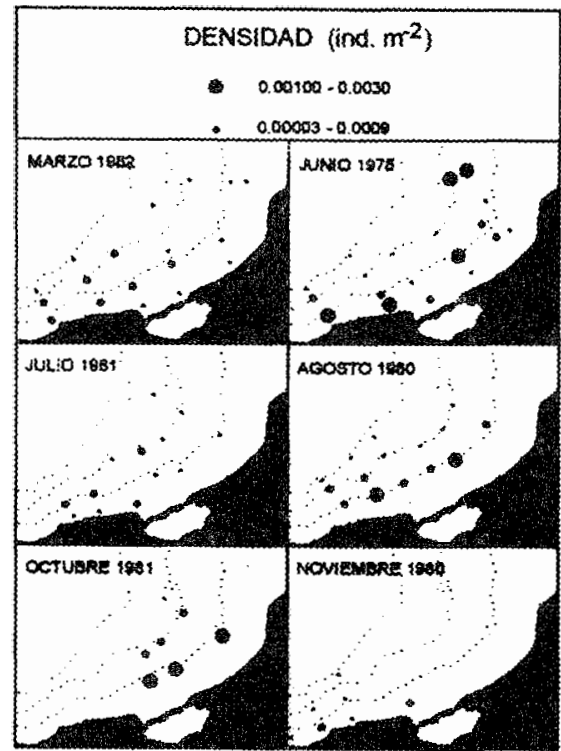

(a)

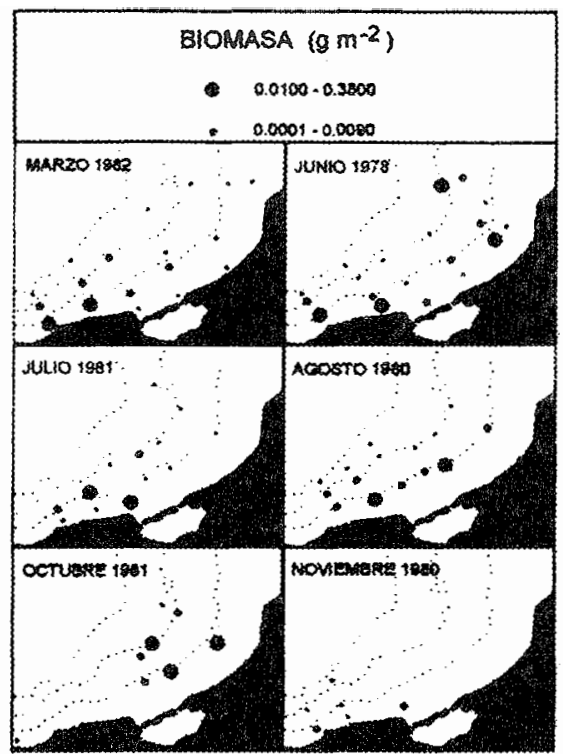

(b)

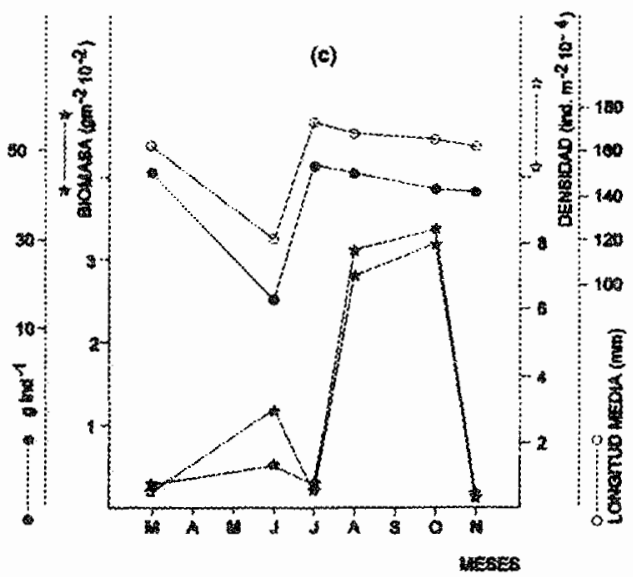

Fig. 3. a) Distribución de la densidad de $O$. oglinum en la Sonda de Campeche en los diferentes meses analizados. b) Distribución de la biomasa. c) Comportamiento estacional de la densidad, biomasa, longitud promedio y peso promedio. 
No se observó una relación evidente entre distribución por tallas y las condiciones estuarinas y batimétricas de la plataforma, encontrándose distribuidas homogéneamente entre los 12 y $36 \mathrm{~m}$ de profundidad (Fig. 2). Sin embargo, esto no excluye que la especie pueda ser encontrada a profundidades menores donde no fue posible un muestreo debido a las características de las embarcaciones e incluso dentro de la Laguna de Términos. En éste ecosistema $O$. oglinum ha sido observada esporádicamente en los sistemas fluvio-lagunares en lluvias (44 a $140 \mathrm{~mm}$ de longitud total) y nortes ( 84 a $149 \mathrm{~mm}$ de longitud total), y ocasionalmente en el litoral interno de la Isla del Carmen y Boca de Puerto Real (Álvarez-Guillén et al. 1985). Asimismo, otros autores en el norte del Golfo de México, Springer y Bullis (1956), Turner y Johnson (1973), y Darnell et al. (1983) han reportado a O. oglinum como especie típica de aguas someras, plataformas insulares y de deltas de ríos, estuarios y bahías. Houde (1977) la reporta como una especie costera abundante en profundidades menores de $35 \mathrm{~m}$, aunque esporádicamente se le encuentra a más de 90 m. Sogard et al. (1989) establecen que $O$. oglinum es una especie de gran actividad nocturna en bancos de pastos marinos. Esto concuerda con el patrón de distribución observado en este estudio al apoyar que es una especie de profundidades someras, que utiliza los sistemas lagunares estuarinos en algún momento de su ciclo de vida.
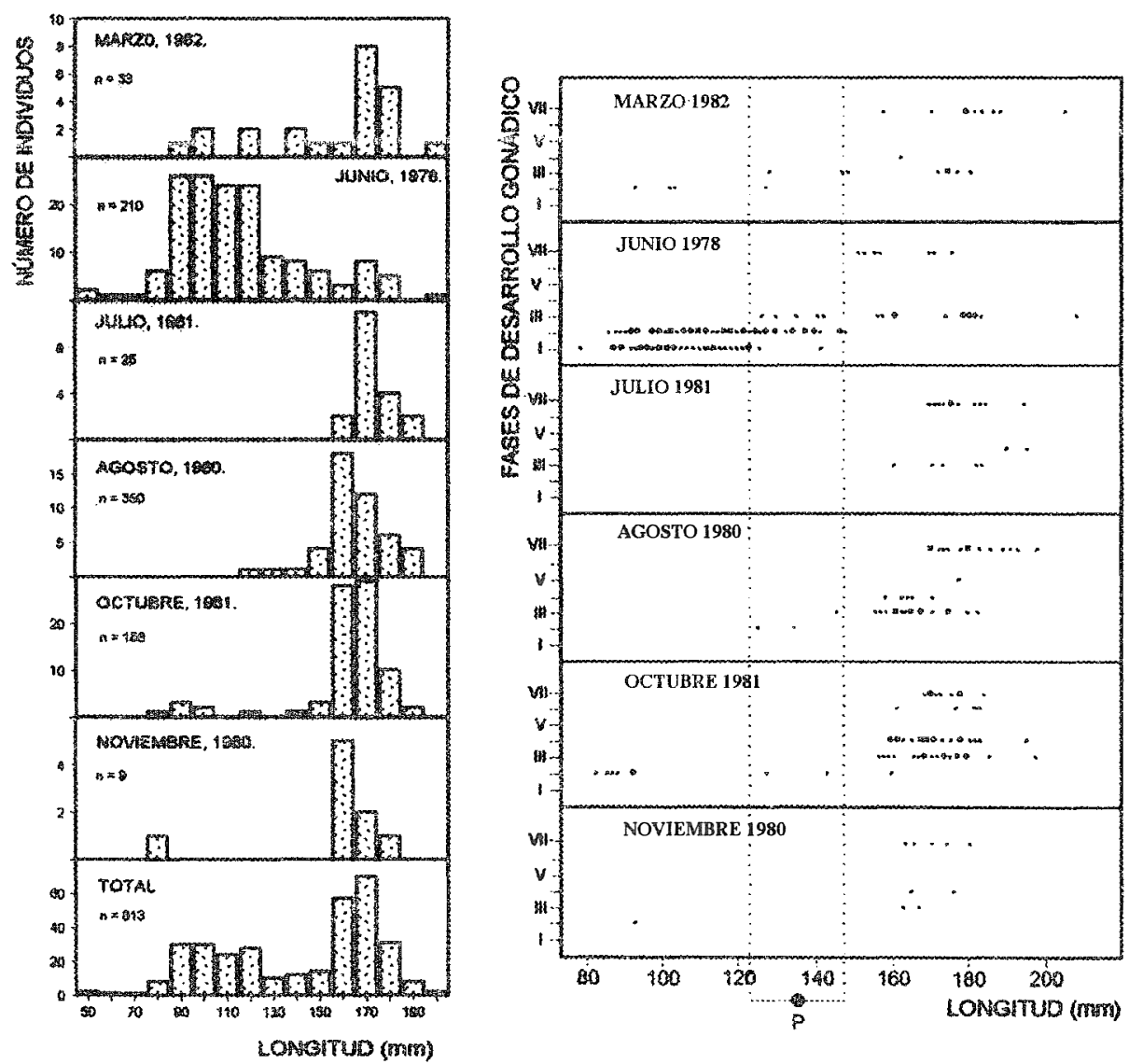

Fig. 4. a) Distribución de frecuencia de tallas y b) relación de la longitud con las fases de madurez gonádica, indicándose la talla de primera madurez $(\mathrm{P})$. 
Reproducción, maduración y crianza: Durante todos los meses, excepto agosto, el número de hembras fue mayor que el de machos (Fig. 5). En marzo la proporción hembras: machos fue de $2: 1$, en junio 1.4: 1 , en julio 3: 1, en agosto $1: 1.3$, en octubre $2: 1$ y en noviembre 2: 1 . Esto podría estar representando una estrategia adaptativa de la especie para tener un mayor stock de huevos y por lo tanto un mayor reclutamiento de juveniles. Reintjes (1980) menciona que se ha observado una fluctuación cíclica en estas proporciones de marzo a diciembre. Por otra parte, únicamente en el mes de junio es donde se observaron individuos sexualmente indeterminados.

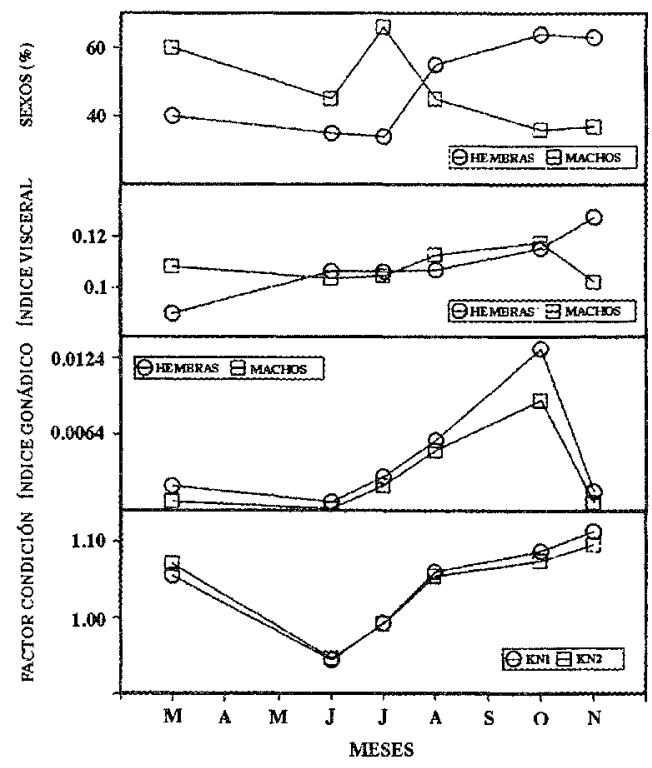

Fig. 5. Comportamiento de la proporción de sexos, índice visceral, índice gonádico y factor de condición $\mathrm{KN}_{1}$ y $\mathrm{KN}_{2}$.

La maduración de $O$. oglinum se presenta a partir de una longitud de $126 \mathrm{~mm}$, con una talia de primera madurez de $135 \mathrm{~mm}$ (Fig. 4). Como puede observarse, las tallas de traslape entre individuos juveniles inmaduros e individuos en maduración fueron de 126 a $145 \mathrm{~mm}$ en el mes de junio por lo que el coeficiente me- dio estimado fue de $135 \mathrm{~mm}$. La presencia de organismos indeterminados sexualmente en junio indica el reclutamiento de juveniles en este mes y un período de reproducción probablemente desde abril y mayo. En agosto se observan individuos de tallas grandes en descanso y en reproducción, previo a un reclutamiento de juveniles durante octubre en donde además se encuentran organismos desovados. Los individuos juveniles más pequeños y las hembras en reproducción y desovados se encontraron distribuidos ampliamente en la región somera de la Sonda de Campeche, tanto en la zona A como en la B. Por esto, no se observa una predilección de área más específica de $O$. oglinum como zona reproductiva. A este respecto, Houde (1977) observa que el desove ocurre en mar abierto, por todo el Golfo de Florida hasta los $50 \mathrm{~m}$ de profundidad y en mayor abundancia de los 10 a los $30 \mathrm{~m}$. Asimismo, ha encontrado huevos desde mayo a agosto y larvas desde marzo a septiembre proponiendo un pico de desove desde mayo a julio. Reintjes (1980) tampoco ha observado juveniles en grandes números, en áreas someras cercanas a la costa. Fuss et al. (1969) sugieren un período de desove de marzo-agosto con un pico notable en junio para Florida y según Hildebrand (1963) la especie tiene un periodo de desove durante mayo-junio en Carolina del Norte. Con esto se puede observar que efectivamente la especie comienza a reproducirse durante la época de secas y que el reclutamiento máximo se presenta en la época de lluvias, utilizando el área somera de la Sonda de Campeche.

Las variaciones del índice visceral y del índice gonádico muestran que los valores crecientes de marzo a octubre parecen estar determinados por el creciente tamaño de las gónadas indicando poca actividad alimentaria. El pico más alto del índice visceral en noviembre corresponde a un bajo valor gonádico. Esto indica que el mayor peso visceral no está determinado por las gónadas, de donde se puede inferir que los individuos juveniles y en descanso respectivamente, se están alimentando activamente desde junio a noviembre (Fig. 5). 
El comportamiento del índice gonádico concuerda con el segundo pico reproductivo y de reclutamiento observados. A partir de julio se observa un aumento de este índice debido al desarrollo gonádico, hasta alcanzar el valor máximo en octubre cuando la especie se está reproduciendo y baja hacia noviembre cuando se presenta el reclutamiento. El otro pico reproductivo y de reclutamiento, se puede inferir de los valores gonádicos más bajos de junio caracterizado por el reclutamiento de juveniles (Fig. 5).

Los valores de condición obtenidos también apoyan este planteamiento (Fig. 5). Entre julio y noviembre coinciden con el largo período de maduración gonádica y el desarrollo somático. El valor más bajo coincide con la presencia de un gran número de juveniles en el mes de junio, en los cuales el desarrollo somático es aún muy reducido y el valor de condición más alto se observa en noviembre después del desove. Hale (1984) en un estudio sobre cantidad de ácidos grasos en esta especie, encontró que hay un decrecimiento del contenido de grasas desde primavera hasta finales del verano, lo que apoya que la especie presenta un desarrollo somático propio de su maduración durante este período y poca acumulación de grasa.

\section{DISCUSIÓN}

Patrón biológico y ecológico: El análisis de la dinámica ecológica de $O$. oglinum, tanto espacial como temporalmente dentro del sistema estuario-plataforma con relación a sus variaciones de distribución y abundancia, rango de tallas, madurez gonádica y reclutamiento, determina un patrón biológico y ecológico que refleja la dinámica ecológica del sistema. En la Fig. 6 se esquematiza a grandes rasgos la exitosa estrategia biológica y ecológica de esta especie que ha permitido que sea dominante entre las casi 300 especies que integran la comunidad de peces demersales en la Sonda de Campeche. Las estrategias adaptativas de las especies de peces están directamente relacio-

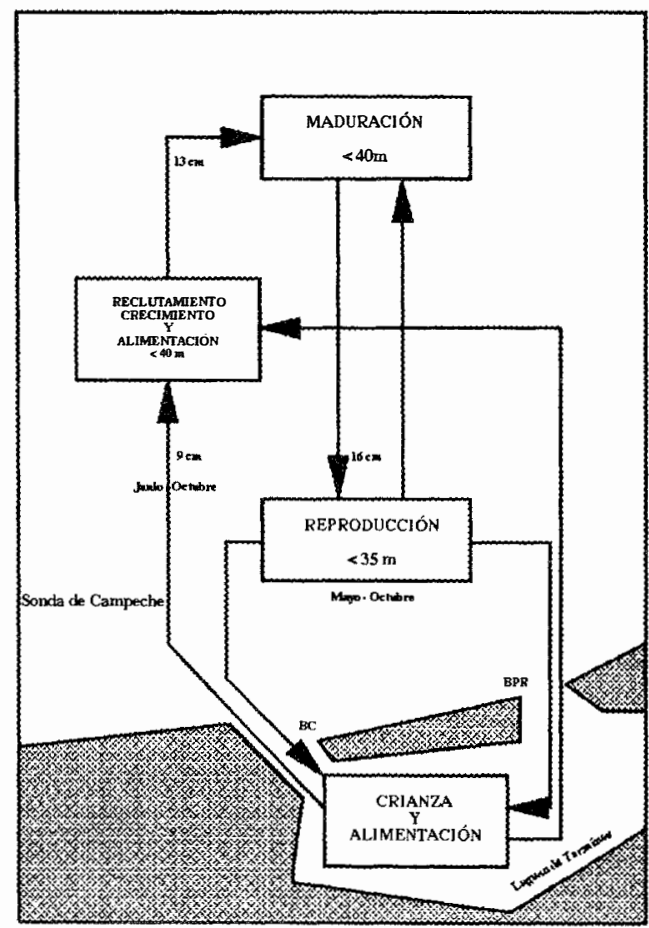

Fig. 6. Patrón biológico de $O$. oglinum donde se indica espacial y temporalmente la reproducción, maduración y crian$\mathrm{za}, \mathrm{y}$ las tallas promedio en las que se presenta cada etapa.

nadas con sus necesidades biológicas a nivel ecosistema, por lo que están en función de la utilización de los procesos ecológicos característicos de la región. $O$. oglinum explota y optimiza los procesos ecológicos típicos de esta región como son la alta productividad y el alto aporte de materia orgánica de los sistemas lagunares-estuarinos, además de la gran diversidad de áreas de alimentación, reproducción, protección, reclutamiento y crianza que estos sistemas representan (Yáñez-Arancibia y Sánchez-Gil 1988b).

Así, se puede establecer que $O$. oglinum se distribuye en los dos subsistemas ecológicos de la Sonda de Campeche a profundidades menores de $36 \mathrm{~m}$. Es una especie dependiente estuarina que eventualmente durante su etapa juvenil utiliza la Laguna de Términos y sistemas estuarinos adyacentes para protegerse, criarse 
y/o alimentarse, y posteriormente se recluta a la población adulta en la Sonda de Campeche. Las hembras maduran a partir de una longitud de $126 \mathrm{~mm}$, con una talla de primera madurez de $135 \mathrm{~mm}$. Presenta un largo período de reproducción en el año, de mayo a octubre, con dos picos máximos en mayo y agosto; y en la región somera (12-36 m) de la Sonda de Campeche. Se presentan dos pulsos marcados de reclutamiento de juveniles en los meses de junio y octubre, relacionados con los pulsos de máxima abundancia. No se observa relación entre la distribución de tallas de los individuos de esta especie y la batimetría, todas se encontraron homogéneamente distribuidas entre los 12 y $36 \mathrm{~m}$ de profundidad, aunque esto no excluye que se le pueda encontrar a profundidades menores. Las máximas abundancias están correlacionadas con los períodos de reclutamiento. La condición de la población es baja durante el primer reclutamiento y alta durante el segundo reclutamiento, debido a la gran cantidad de organismos de tallas pequeñas en junio y su escaso desarrollo somático.

\section{AGRADECIMIENTOS}

Se agradece al Instituto de Ciencias del Mar y Limnología de la Universidad Nacional Autónoma de México y al Programa EPOMEX de la Universidad Autónoma de Campeche, el apoyo institucional y económico para la realización del presente estudio. El trabajo formó parte de los proyectos de investigación "Ecología y evaluación de recursos demersales marinos" (UNAM-CONACYT, clave PCCNCNA050815) y "La pesca y los recursos pesqueros críticos del Estado de Campeche" (UAC-EPOMEX-SEP, 1990).

\section{RESUMEN}

Se analizan diversos aspectos de la biología y ecología de Opisthonema oglinum con base en 813 individuos obtenidos en seis cruceros oceanográficos. Esta especie dependiente estuarina, se distribuye en la Sonda de Campeche entre los 12 y $36 \mathrm{~m}$. Las hembras tienen una talla de primera madurez de $135 \mathrm{~mm}$ de LT. Se reproduce desde mayo a octubre, con dos máximos en mayo y agosto, a profundidades mayores de $18 \mathrm{~m}$. Los juveniles se encuentran en la plataforma y dentro de la Laguna de Términos. El reclutamiento se presenta en junio y octubre. Las máximas abundancias se presentan alrededor de las isobatas de 12 y $36 \mathrm{~m}$, y en nortes en el área carbonatada.

\section{REFERENCIAS}

Álvarez-Guillén, H., A. Yáñez-Arancibia \& A.L. Lara Domínguez. 1985. Ecología de la Boca del Carmen, Laguna de Términos: El hábitat y estructura de las comunidades de peces. An. Inst. Cien. del Mar y Limnol. Univ. Nal. Autón. México 12: 107-144.

Cailliet, G.M., M.S. Love \& A.W. Ebeling. 1986. Fishes. A field and laboratory manual on their structure, identification, and natural history. Wadsworth Publishing Company, Belmont, California. 194 p.

Carranza-Edwards, A., L. Rosales-Hoz \& A. Monreal-Gómez. 1993. Suspended sediments in the southeastern Gulf of Mexico. Mar. Geol. 112: 257-269.

Darnell, R.M., R.E. Defenbaugh \& D. Moore. 1983. Northwestern gulf shelf bio-atlas; a study of the distribution of demersal fishes and penaeid shrimp of soft bottoms of the continental shelf from the Rio Grande to the Mississippi river delta. Open File Report No. 82-04. Metaire, LA: Minerals Management Service, Gulf of Mexico OCS Regional Office. 438 p.

Darnell, R.M. \& J.A. Kleypas. 1987. A study of the distribution of demersal fishes and penaeid shrimp of soft bottoms of the continental shelf from the Mississippi river delta to the Florida Keys. OCS STUDY MMS 86-0041.

Fuss, C.M.Jr., J.A. Kelly Jr. \& K.W. Prest Jr. 1969. Gulf thread herring: aspects of the developing fishery and biological research. Proc. Gulf Caribb. Fish. Inst. 21: 111-125.

Grant, C.J. 1981. Gill net selectivity and catch rates of coastal pelagic fish in Jamaica. Estuar. Coast. Shelf. Sci. 12: 167-175.

Hale, M.B. 1984. Proximate chemical composition and fatty acids of three small coastal pelagic species. Mar. Fish. Rev. 46: 19-21. 
Harvey, G.C.McN. 1980. Preliminary studies of the coastal pelagic fishes of Jamaica. Proc. Assoc. Isl. Mar. Lab. Caribb. 15: 24.

Hildebrand, S.F. 1963. Family Clupeidae, p 257-454. In H.B. Bigelow (ed.). Fishes of the western North Atlantic. Part Three. Mem. Sears Found. Mar. Res. Yale University, Coneticut.

Houde, E. D. 1977. Abundance and potencial yield of the Atlantic thread herring, Opisthonema oglinum, and aspects of its early life history in the eastern Gulf of Mexico. Fish. Bull. 75: 493-512.

Houde, E.D., Ch. Grall \& St.A. Berkeley. 1983. Population parameter estimates for three shoaling pelagic fishes in the eastern Gulf of Mexico. Ices Council Meeting, Copenhague, Dinamarca. $12 \mathrm{p}$

Laevastu, T. 1971. Manual de métodos de biología pesquera. Publicación FAO. Acribia, Zaragoza. 243 p.

LeCren, E.D. 1951. The length-weight relationships and seasonal cycle in the perch (Percafluviatilis). J. Anim. Ecol. 20: 201-219.

Ogawa, M. \& P. Barreto. 1979. Industrial Utilization of Small-Sized Fishes in Northeastern Brazil. Bol. Cienc Mar. 33: 11

Pauly, D. 1982. Studying single-species dynamics in a tropical context, p. 33-70. In D. Pauly, D. \& G.I. Murphy (eds.). Theory and management of tropical fisheries. ICLARM Conference Proceedings 9, Manila, Filipinas.

Pauly, D. \& A. Yáñez-Arancibia. 1994. Fisheries in Coastal Lagoons, p 377-399. In B. Kjerfve (ed.). Coastal Lagoon Processes. Elsevier Oceanography Series, Amsterdan, Holanda.

Reintjes, J. W. 1980. Marine herring and sardine resources of the northern Gulf of Mexico. In M. Flandorfer and L. Skuplen (ed.) Proceedings of a Workshop for Potential Fishery Resources of the Northern Gulf of Mexico. Nueva Orleans. Publ. No. MASGP-80-012.

Sogard, S.M., G.V. Powell \& J.G. Holmquist. 1989. Utilization by fishes of shallow, seagrass-covered banks in Florida Bay: 2. Diel and tidal patterns. Environ. Biol. Fish. 24: 81-92.

Springer, S. \& H.R. Bullis Jr. 1956. Collections by the Oregon in the Gulf of Mexico. U. S. Fish. and Wild. Serv. Spec. Sci. Rept. 196: 1-134.
Tapia-García, M., A. Yáñez-Arancibia, P. Sánchez-Gil \& M.C. García-Abad. 1995. Distribución, abundancia y reproducción de Priacanthus arenatus Cuvier (Pisces: Priacanthidae), en la plataforma continental del sur del Golfo de México. Biotropica 27: 232-237

Turner, W.R. \& G.N. Johnson. 1973. Distribution and relative abundance of fishes in New Port River, North Carolina. NOAA Tech. Rep. NMFS-SSRF-666: 1-23.

Valdés, E. \& M.C. Sotolongo. 1983. Algunos aspectos de la biología y pesquería del machuelo (Opisthonema oglinum) de la plataforma suroriental cubana. Rev Cub. Invest. Pesq. 8: 66-98.

Vasconcelos, F.A.L. 1979. Estudo Ecologico da Regiao de Itamaraca, Pernambuco, Brasil. 4. Alimentacao da Sardinha Bandeira, Opisthonema oglinum (Le Sueur 1817), no Canal de Santa Cruz. Trab. Oceanogr. Univ. Fed. Pernambuco. 14: 105-116.

Yáñez-Arancibia, A. \& P. Sánchez-Gil. 1986. Los peces demersales de la plataforma continental del sur de Golfo de México. Vol. 1. Caracterización del ecosistema y ecología de las especies, poblaçiones y comunidades. Inst. Cienc. del Mar y Limnol. Univ. Nal. Autón. México. Publ. Esp. 9: 229 p.

Yáñez-Arancibia, A. \& P. Sánchez-Gil. 1988a. Caracterización ambiental de la Sonda de Campeche frente a la Laguna de Términos, p. 41-50. In A. Yáñez-Arancibia \& J.W. Day, Jr. (eds.). Ecología de los ecosistemas costeros en el sur del Golfo de México: La región de la Laguna de Términos. Inst. Cienc. del Mar y Limnol. UNAM, Coast. Ecol. Inst. LSU. Universitaria, México, D.F.

Yáñez-Arancibia, A. \& P. Sánchez-Gil. 1988b. Ecología de los recursos demersales marinos: Fundamentos en costas tropicales. AGT, México, D. F. 228 p.

Yáñez-Arancibia, A., A. L. Lara-Domínguez \& J. W. Day Jr. 1993. Interaction between mangrove and seagrasses habitat mediated by estuarine nekton assemblages: coupling of primary and secondary production. Hidrobiologia 264: 1-12

Yáñez-Arancibia, A., A. L. Lara-Domínguez \& D. Pauly. 1994. Coastal lagoons as fish habitats, p. 363-376. In B. Kjerfve (ed.). Coastal Lagoon Processes. Elsevier Oceanography Series, Amsterdan, Holanda. 